\title{
Aspectos da avaliação de pulverizadores hidráulicos de barra na região de Uberlândia, Minas Gerais
}

\author{
Aspects of boom hydraulic sprayer evaluation in the region of Uberlândia, \\ Minas Gerais
}

Cleyton Batista de Alvarenga ${ }^{1}$, João Paulo Arantes Rodrigues da Cunha ${ }^{2}$, Mauri Martins Teixeira ${ }^{3}$

\begin{abstract}
RESUMO
A avaliação de pulverizadores é fundamental para melhorar a tecnologia de aplicação de agrotóxicos. O objetivo deste trabalho foi avaliar os aspectos quantitativos do estado de funcionamento de pulverizadores agrícolas na região de Uberlândia, Minas Gerais, e a metodologia de inspeção para as condições brasileiras. As avaliações foram realizadas em propriedades rurais visitadas aleatoriamente e consistiram de um questionário preenchido pelo operador e/proprietário das máquinas e da inspeção propriamente dita dos pulverizadores. Os itens avaliados foram: distribuição volumétrica na barra, rotação na tomada de potência, volume de aplicação, vazão das pontas de pulverização, espaçamento entre bicos, nível de ruído e exatidão do manômetro. De acordo com os resultados, a inspeção evidenciou a necessidade da implantação de um programa de avaliação freqüente desses equipamentos, visto que a manutenção dos pulverizadores hidráulicos de barra está sendo feita de forma errônea. A metodologia utilizada foi adequada para a avaliação, exceto para uniformidade de distribuição volumétrica na barra.
\end{abstract}

Palavras-chave: tecnologia de aplicação, aplicação de defensivos, inspeção de máquinas agrícolas.

\section{ABSTRACT}

Sprayer evaluation is an important tool to improve pesticide application technology. The objective of this study was to evaluate the quantitative aspects of the working conditions of boom sprayers in the region of Uberlândia, Minas Gerais, and the inspection methodology for Brazilian conditions. The evaluations were done in farm properties randomly visited and consisted of a questionnaire filled out by the equipment operator and by the inspection team during in loco visits. The items evaluated were: boom volumetric distribution, rotation at power take-off, spray volume, flow rate at the nozzle tips, spacing between nozzles, noise level and manometer precision. The results indicated the need to implement a frequent evaluation program for this equipment, since boom maintenance is being done incorrectly. The method used was adequate to evaluate the working conditions of the agricultural boom sprayers, except for boom volumetric distribution.

Key words: spray technology, pesticide application, agricultural machinery inspection.

\section{Introdução}

O conhecimento do estado atual dos pulverizadores agrícolas no Brasil é fundamental para melhorar a tecnologia de aplicação dos agroquímicos, principalmente na região dos Cerrados, onde a agricultura é praticada intensivamente. Além disso, as exigências de programas de certificação da produção agrícola têm crescido, principalmente, para produtos de exportação. Em breve, muitos produtos exportados, precisarão de laudo de qualidade, o qual deverá constar também a descrição das condições das máquinas responsáveis pela aplicação dos agroquímicos.
Neste contexto, insere-se a região do Triângulo Mineiro, com sua agricultura entre as mais avançadas do Brasil em termos de produtividade. No entanto, em termos de qualidade na aplicação de agrotóxicos e impactos ambientais, pouca informação há a respeito.

Uma medida adotada em vários países da Europa para controlar a qualidade das aplicações é a realização de inspeções periódicas dos pulverizadores, atribuindo a eles certificação. É oferecida orientação aos produtores para um melhor uso e manutenção dos equipamentos, objetivando redução da quantidade de defensivos agrícolas utilizados, com conseqüente redução do custo e da contaminação ambiental, além

1 Doutorando Departamento de Engenharia Agrícola, Universidade Federal de Viçosa. Av. Ph Rolfs s/n secretaria de PósGraduação, 36570-000, Viçosa-MG, Cleyton.alvarenga@ufv.br.

2 Professor Dr. Instituto de Ciências agrarias, Universidade Federal de Uberlândia, jpcunha@ iciag.ufu.br

3 Professor Dr. Departamento de Engenharia Agrícola, Universidade Federal de Viçosa, mauri@ufv.br.

Fecha de Recepción: 14 Julio, 2009.

Fecha de Aceptación: 13 Enero, 2011. 
de elevar a eficiência das aplicações (Gandolfo \& Antuniassi, 2003).

Na Alemanha, Koch (1996) propôs uma metodologia de avaliação em pulverizadores dividindo-as em qualitativas (observadas) e quantitativas (medidas). Dentre as quantitativas, apresenta-se a avaliação da vazão das pontas, comprimento real da barra, velocidade de trabalho entre outras.

No Centro de Testes de Pordenone, na Itália, a cada ano, cerca de 100 pulverizadores são inspecionados na região produtora de frutas. Por volta de $39 \%$ e $11 \%$, respectivamente, da área total dos pomares (700 ha) e de vinhas (5500 ha), em torno deste distrito, são atualmente tratados com pulverizadores que foram inspecionados ao menos uma vez durante os últimos cinco anos (Pergher, 2004).

Atualmente, a ideia de inspeção de pulverizadores no Brasil ainda é incipiente. Algumas universidades e empresas privadas estão iniciando projetos de inspeção, em fase experimental, sem qualquer oficialização. Acredita-se, entretanto, que a tendência futura é a obrigatoriedade das avaliações. Dessa forma, o país precisa de informações sobre este processo que subsidiem a criação de um programa de inspeção eficaz, robusto e viável economicamente. $\mathrm{O}$ problema para se atingir resultados satisfatórios no controle das aplicações de agroquímicos é a dificuldade de se dominar todos os fatores que interagem e influenciam no processo de pulverização (Santos \& Maciel, 2006).

Analisando a presença e estado dos principais componentes essenciais para o uso dos pulverizadores agrícolas, Silveira et al. (2006), na região de Cascavel no Paraná, constataram que somente 17\% dos equipamentos estavam em condições adequadas de uso, evidenciando a necessidade da adoção de um sistema oficial de inspeção de pulverizadores agrícolas. Borghi et al. (2003), avaliando a qualidade de pulverizadores em pequenas propriedades, constataram que o coeficiente de variação das pontas ensaiadas para a distribuição dos agroquímicos atingiram índices superiores aos estabelecidos pela Organização das Nações Unidas para Agricultura e Alimentação (FAO), que preconiza coeficiente de variação inferior a $10 \%$.

Desta forma, o objetivo deste trabalho foi avaliar os aspectos quantitativos do estado de funcionamento de pulverizadores agrícolas na região de Uberlândia, Minas Gerais, e a metodologia de inspeção para as condições brasileiras.

\section{Material e Métodos}

O trabalho foi realizado na região em Uberlândia, Minas Gerais. Trata-se de uma área do Cerrado Brasileiro, caracterizada por médias e grandes propriedades produtoras de grãos. Foram visitadas 26 propriedades rurais, escolhidas aleatoriamente, que possuíam pulverizadores hidráulicos de barra.

Inicialmente, foi montada uma estrutura auxiliar para as avaliações, bem como definida a metodologia de análise. Para os levantamentos, utilizou-se um kit de avaliação, composto por: bomba de calibração com manômetro padrão digital (Classe A4), para aferição da exatidão dos manômetros; tacômetro Minipa modelo MDT-2238A (foto/contato digital), para aferição da rotação na tomada de potência, provetas graduadas; cronômetro e máquina fotográfica digital. Após a descrição geral do equipamento, conforme proposto por Koch (1996), as avaliações foram realizadas do ponto de vista quantitativo sobre parâmetros de prestação. Buscou-se empregar metodologia simples e barata para facilitar sua implementação futura.

A regularidade do espaçamento entre bicos foi determinada em todos os pulverizadores avaliados. Os valores foram tomados com auxílio de uma trena com resolução de um milímetro. A máquina foi considerada ideal, quando todos os espaçamentos foram iguais. Variações de até 10\% na distância entre bicos foram considerados aceitáveis; já variações maiores que $10 \%$ foram considerados inadequados para a aplicação de agroquímicos.

A exatidão dos manômetros foi aferida após as avaliações dinâmicas, com a instalação do manômetro do pulverizador na bomba de calibração. Esta bomba hidráulica é composta por um manômetro de precisão, instalado em uma tubulação comunicante com o bocal de instalação do manômetro da máquina, sendo a tubulação ligada a um cilindro contendo um êmbolo. Este êmbolo é acionado pelo movimento obtido pelo acionamento manual de uma rosca sem-fim. O movimento da rosca, bem como a posição do êmbolo, determina o nível de pressurização de água dentro do cilindro, comunicando a pressão com ambos os manômetros, possibilitando comparações entre ambos. Conforme previsto na norma NBR-12446/1992, as comparações foram realizadas com pressão mínima de $25 \%$ da escala máxima até o valor máximo correspondente a $75 \%$ do fundo de escala. Foram feitas, no mínimo, cinco leituras neste intervalo no sentido ascendente e 
descendente. Tais componentes foram considerados exatos, quando as diferenças entre as leituras correspondentes do manômetro da máquina e o padrão não superaram $10 \%$ de variação.

A aferição do volume de aplicação foi realizada somente nas máquinas que estavam calibradas. Os valores informados pelos usuários foram comparados com os valores encontrados pela equipe de avaliação e, pela diferença entre ambos, foi obtido um valor correspondente ao erro no volume de aplicação. O limite de erro para que a calibração fosse considerada aceitável foi de 5\%. Para tanto, analisaram-se: velocidade de trabalho, faixa de aplicação e vazão na barra, de acordo com as condições empregadas pelo operador da máquina.

Para a determinação da uniformidade de distribuição volumétrica na barra, buscou-se uma metodologia alternativa às mesas de distribuição. Para isto, utilizaram-se dez provetas iguais, com capacidade de um litro e resolução de cinco mililitros. As provetas foram colocadas uma ao lado da outra e alinhadas sob a barra, na altura de trabalho adotada pelo operador no momento das aplicações, que na maioria das propriedades foi de $50 \mathrm{~cm}$. Feito isso, o pulverizador era acionado, na pressão normal de trabalho empregada pelo operador, por um tempo suficiente para uma leitura mínima nas provetas de 100 mililitros, sendo que a coleta de liquido foi realizada em três pontos amostrais por barra. Para encontrar o coeficiente de variação (CV), utilizaram-se as médias das leituras das provetas, por meio da equação utilizada por Freitas (2005) e Bauer et al., (2006). Coeficiente de variação da média dos volumes das provetas de até $10 \%$ foi considerado satisfatório. Entre $10 \%$ e $15 \%$, foram considerados aceitáveis. Já para coeficientes acima de $15 \%$, inadequado. Para a realização deste teste, em virtude da falta de um galpão fechado na maioria das propriedades com disponibilidade para a abertura completa das barras, buscaram-se condições propícias de ambiente, como temperatura inferior a $30{ }^{\circ} \mathrm{C}$, umidade relativa superior a $60 \%$ e velocidade do vento inferior a $10 \mathrm{~km} \mathrm{~h}^{-1}$. No entanto, devido ao horário, disponibilizado aos avaliadores para checagem das máquinas, nem sempre foi possível obter essas condições ótimas, o que influenciou as avaliações.

A vazão das pontas foi avaliada com auxílio de provetas graduadas, com capacidade de um litro e resolução de cinco mililitros. Coletou-se o líquido passados três minutos de funcionamento para estabilização da pressão no circuito hidráulico. A pressão avaliada foi aquela usada pelo operador na condição de regulagem do equipamento. Posteriormente, fez-se a comparação das vazões de cada ponta com a média de todas as pontas avaliadas. As pontas foram consideradas ruins ou inadequadas para uso, quando a diferença percentual entre sua vazão e a média diferiu em mais de $10 \%$.

A rotação na tomada de potência dos tratores, para os pulverizadores tratorizados, foi determinada com o auxilio de um tacômetro. A rotação considerada ideal foi de $540 \mathrm{rpm}$ no sentido horário. Para fins de avaliação, foram considerados aceitáveis para funcionamento aqueles tratores que apresentaram uma variação de mais ou menos $10 \mathrm{rpm}$ na tomada de potência (Mialhe, 1996). Esta avaliação foi realizada de acordo com as condições de trabalho executada pelos operadores na propriedade.

As avaliações do nível de ruído basearam-se no método descrito na NBR-9999 (Abnt, 1987). Segundo essa norma, na posição e momento do ensaio de medição do nível de ruído, a temperatura ambiente deve estar entre -5 e $30^{\circ} \mathrm{C}$ e a velocidade do vento deve ser inferior a $5,0 \mathrm{~m} \cdot \mathrm{s}^{-1}$. Os níveis de ruído foram determinados em um medidor de pressão sonora (decibelímetro), nos circuitos de resposta lenta e de equalização "A", sendo expressos em $\mathrm{dB}(\mathrm{A})$.

Embora tenham sido observadas condições climáticas favoráveis, durante a realização de todos os testes, foi utilizado o protetor de ventos no microfone do medidor de pressão sonora, com o intuito de uniformizar as condições de leitura e evitar a influência de possíveis rajadas de vento. As leituras foram feitas junto ao ouvido do operador, no lado de maior intensidade sonora, em períodos de dez segundos, perfazendo cinco leituras para cada condição. Em todas as amostragens realizadas, foram aguardados três minutos antes da medição, tempo necessário para que as máquinas entrassem em regime de trabalho. Para a medição, os pulverizadores e tratores foram acionados e colocados na condição de operação normal, com rotação no motor do trator equivalente a rotação de $540 \mathrm{rpm}$ na tomada de potência.

Além desses itens quantitativos, por intermédio de questionário aplicado junto ao operador ou proprietário da máquina e pela observação realizada nos pulverizadores pela equipe de avaliação, foi verificado seu ano de fabricação, quando possível. Essa informação forneceu uma idéia da idade da 
frota de pulverizadores, na região de Uberlândia, e foi apontada, em alguns casos pelos operadores, como uma justificativa para problemas apresentados.

\section{Resultados e Discussão}

Foram avaliados 34 pulverizadores de barra em 26 propriedades agrícolas. Adotaram-se os conceitos de Koch (1996), para apresentação dos resultados quantitativos. Estes são aqueles parâmetros mensurados por aparelhos considerados como padrão ou medidos por equipamentos específicos para tal finalidade, e também o volume de aplicação. Nas Figuras 1, 2 e 3, são apresentados os resultados obtidos a partir das leituras feitas nas máquinas e extrapolados para seus limites de tolerância, acima e/ou abaixo dos quais os danos ao equipamento e os prejuízos foram considerados inaceitáveis e exigem reparos.

A análise da vazão das pontas de pulverização (Figura 1) mostrou que 32\% das máquinas apresentaram ao menos uma ponta funcionando com vazão diferindo em relação à média em mais de $10 \%$. O equipamento que apresentou maior número de pontas com vazão $10 \%$ abaixo da média tinha quatro pontas nessa condição e o menor, uma ponta. Já para vazão acima da média, o maior número de pontas em um único equipamento foi cinco e o menor, duas pontas. Os maiores erros ocorreram nas pontas que estavam acima da média.

As causas principais estão ligadas a pontas entupidas ou desgastadas. Vários operadores não sabiam ou não se lembravam quando haviam sido trocadas as pontas, o que levanta a hipótese de estas pontas estarem sendo usadas por muitas safras sem serem trocadas ou até mesmo, em alguns equipamentos, serem originais de fábrica. Todo operador pode fazer um teste para analisar a vazão das pontas na propriedade, basta ter a disposição um vasilhame graduado. Os volumes coletados no mesmo período de tempo entre as pontas podem ser uma indicação do estado de conservação das pontas, auxiliando na tomada de decisão com relação ao momento de substituir as pontas antigas. Outra causa para o alto índice de pontas operando com vazão acima da média, além do tempo de uso, pode ser a limpeza incorreta das pontas com pedaços de arame ou outro material abrasivo que cause dano ao orifício de saída de calda, provocando um maior erro na aplicação de defensivos. Resultados mais preocupantes foram encontrados por Magdalena \& Di Prinzio (1992), que constataram $70 \%$ das máquinas avaliadas com pontas desgastadas. Ramos \& Cortés (2006) encontraram $94 \%$ dos equipamentos com variação de vazão nas pontas, em relação à vazão nominal, superior a 15\%. Segundo Ramos \& Pio (2003), quando três ou mais pontas, num conjunto de 12 , apresentam a vazão alterada em $10 \%$ ou mais, recomenda-se a troca de todo o conjunto.

Com relação à regularidade do espaçamento entre bico como ilustrado na figura 1 , nenhuma máquina apresentou distância entre bicos $10 \%$ abaixo da indicada pelo fabricante, contudo $24 \%$ apresentaram distância entre bicos $10 \%$ acima da indicada. Não houve associação deste problema com a idade das máquinas, ilustrada na figura 2 , o que poderia ser esperado, visto que os equipamentos mais antigos apresentavam tubulações flexíveis entre bicos, como mangueiras, que permitiam aos produtores fazer alterações nesse espaçamento. Não

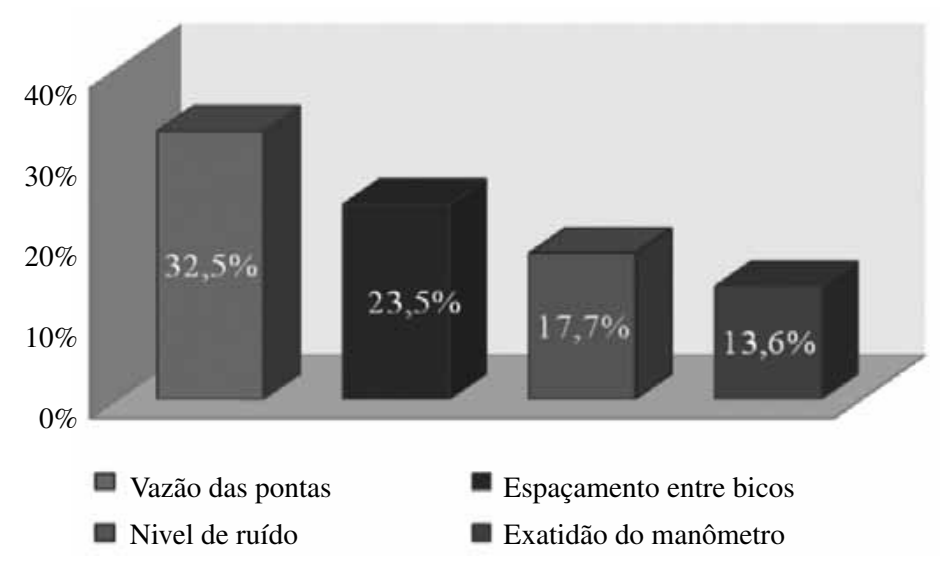

Figura 1. Percentagem de pulverizadores com irregularidades nos parâmetros quantitativos avaliados em pulverizadores de barra na região de Uberlândia. 
se pode afirmar com certeza que estes equipamentos vieram de fábrica com este tipo de irregularidade, pois já tinham no mínimo um ano de trabalho na propriedade, entretanto, como nos pulverizadores mais novos os bicos são fixos, fica a necessidade de um estudo mais detalhado para aferir o distanciamento entre bicos no momento que a máquina sai de fábrica.

A frota da região não pode ser considerada velha, visto que $85,7 \%$ dos equipamentos apresentam até 10 anos de uso e a idade média é de 6,4 anos, sendo, portanto, uma frota relativamente nova. Ramos \& Cortés (2006), avaliando na Espanha o estado funcional de pulverizadores, encontraram que $83 \%$ dos equipamentos apresentavam-se com menos de cinco anos de uso.

A avaliação da acurácia dos manômetros (figura 1) mostrou que um número pequeno de pulverizadores estava operando com leitura do medidor de pressão fora do recomendado, se comparado com resultados apresentados por Gandolfo \& Antuniassi (2003), em que ocorreu problema com a exatidão dos manômetros em $71 \%$ dos pulverizadores. Notase que é desprendida uma atenção maior a esse dispositivo pelos produtores da região, mostrando uma evolução no comportamento do agricultor. Este desgaste no manômetro pode interferir no volume de aplicação e na vazão das pontas. Também não foi constatada relação entre a idade da máquina e a regularidade dos manômetros, ou seja, foram encontrados manômetros com erros de leitura em todas as classes de idade de máquinas, o que pode indicar que alguns manômetros podem vir com defeito da fábrica ou terem uma vida útil muito reduzida.
Os equipamentos autopropelidos ou tracionados por trator cabinado não apresentaram nível de ruído acima de $85 \mathrm{~dB}(\mathrm{~A})$. Todos aqueles que estavam com ruído acima de $85 \mathrm{~dB}$ eram pulverizadores sem cabine no trator. De posse desses resultados, e sabendo da importância e da influência que a exposição por longo período a níveis de ruído acima dos permitidos tem na saúde, recomenda-se que o protetor auricular seja incorporado ao cotidiano dos operadores juntamente com os outros Equipamentos para Proteção Individual (EPIs), sendo fornecidos pelos empregadores como uma medida de proteção e prevenção a problemas de saúde do operador e atendendo à legislação.

É importante destacar que a atenção dentro do processo de pulverização é despendida principalmente para o uso de Equipamentos de Proteção Individual (EPI) voltados à proteção contra exposição ao agrotóxico. Já o nível de ruído não é observado por produtores e operadores, que desconhecem a existência da lei que determina níveis máximos de exposição e os riscos à saúde que estão expostos. A tendência dos fabricantes de máquinas agrícolas em produzir equipamentos autopropelidos e tratores cabinados contribui para reduzir os problemas gerados pela incidência de ruídos encontrada nos tratores sem cabine. Além disso, trazem mais conforto durante a execução dos trabalhos e proteção ao operador da exposição aos produtos que sofrem deriva.

Os resultados mostraram que todos os pulverizadores apresentaram coeficiente de variação (CV) da distribuição volumétrica da barra acima de $15 \%$, como mostra a figura 3 , valor considerado alto e que, portanto, compromete a eficiência da pulverização. Na Itália, Baldi \& Vieri (1992)

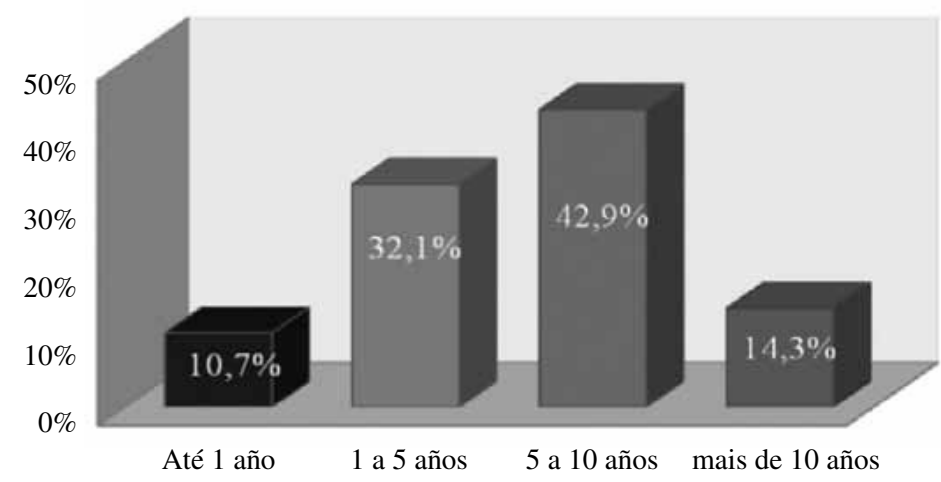

Figura 2. Idade dos pulverizadores avaliados. 
encontraram que $40 \%$ dos pulverizadores estavam com CV na barra acima de $10 \%$. Gandolfo \& Antuniassi (2003) encontraram CV médio de $18,1 \%$, o que é um resultado bom comparado aos encontrados neste trabalho. Entretanto, a metodologia empregada com uso de provetas graduadas não foi considerada adequada, principalmente em virtude da influência do vento nos resultados encontrados. A falta de uniformidade na distribuição volumétrica pode ocorrer devido a vários fatores que compõem o sistema de pulverização de um pulverizador, tais como: mangueiras entupidas e perfuradas; conexões defeituosas e com diâmetros e tamanhos inadequados; pontas entupidas, diferentes, quanto à vazão e ao ângulo, e desgastadas; falta de sistema antigotejo e barras de pulverização desalinhada, deformada e instável. Estes resultados devem ser analisados com critério. Preconiza-se que esse tipo de ensaio seja conduzido em bancada de ensaio padronizada e em condições ambientais propícias. Ou seja, para esta informação ser considerada segura o ensaio deveria ser realizado em condições controladas, preferencialmente em laboratório. A utilização de lonas plásticas e mesas de estudo de deposição poderia minimizar o problema, no entanto, em virtude dos diferentes tamanhos da barra não seria de fácil execução. Vale ressaltar que os procedimentos de inspeção periódica de pulverizadores de barra na Europa, em várias localidades não incluem a avaliação da uniformidade de distribuição volumétrica.

Quanto à rotação na tomada de potência (TDP), $30,4 \%$ dos tratores que estavam sendo empregados na aplicação de defensivos agrícolas, (Figura 3), acoplados a pulverizadores de arrasto e montados, apresentaram valores acima de $550 \mathrm{rpm}$ e 43,5\% estavam com rotação abaixo de $530 \mathrm{rpm}$. A rotação abaixo da recomendada pode ter influências negativas no funcionamento da bomba e no sistema de agitação da calda. Com relação à bomba, poderá afetar sua vazão e também a vida útil. No sistema de agitação mecânica, a rotação baixa afeta o número de revoluções da hélice do agitador. $\mathrm{Na}$ agitação hidráulica, pode afetar o retorno da calda ao tanque. Em ambas, poderá afetar a eficiência dos tratamentos, principalmente devido a entupimentos no circuito hidráulico e formação de espuma acima da calda. Balestrini (2006) verificou que $64 \%$ das bombas apresentaram problemas e que o sistema de agitação não estava funcionando corretamente em $76 \%$ dos equipamentos avaliados, problemas que podem estar relacionados com a rotação na tomada de potência.

A calibração correta dos pulverizadores é pré-requisito para que o controle fitossanitário seja eficiente. Dentre os equipamentos avaliados, (Figura 3), 41,9\% das máquinas estavam aplicando volume de calda abaixo do desejado e $22,6 \%$, acima. Este problema tem um impacto muito grande nos custos com aplicação de agrotóxicos e, conseqüentemente, nos custos de produção da lavoura. Esses resultados mostram que os operadores não estão devidamente preparados para efetuar os cálculos dos volumes aplicados.

De maneira geral, a metodologia empregada na avaliação dos pulverizadores agrícolas na região de Uberlândia mostrou-se adequada, com exceção da avaliação da uniformidade de distribuição

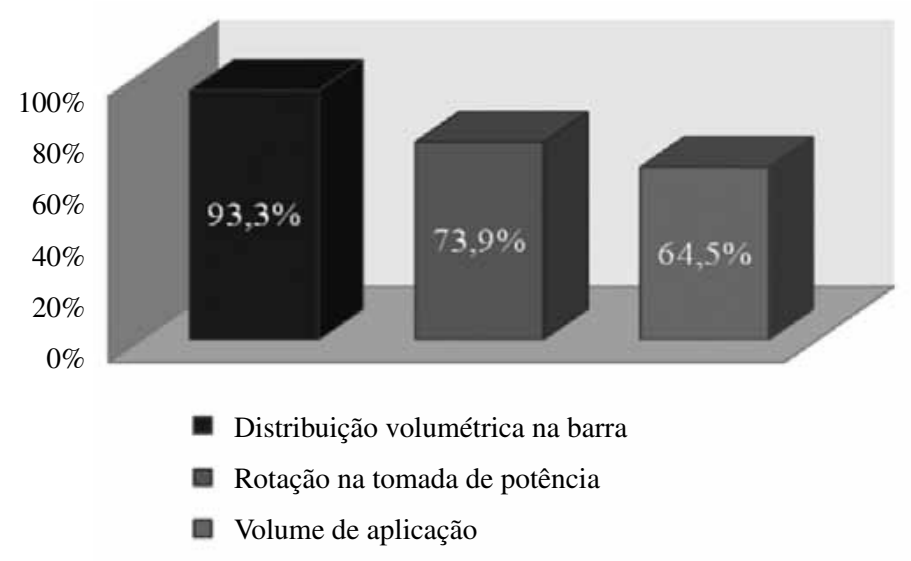

Figura 3. Percentagem de pulverizadores com irregularidades nos parâmetros quantitativos avaliados em pulverizadores de barra na região de Uberlândia. 
volumétrica. A estrutura montada foi simples e de custo relativamente pequeno. $\mathrm{O}$ conjunto de procedimentos alcançou também o objetivo de incentivar os produtores a realizarem avaliações em seus pulverizadores, de forma a obterem informações que auxiliem na manutenção destes equipamentos e melhorem o processo de aplicação de agroquímicos.

\section{Conclusões}

A inspeção dos pulverizadores demonstrou haver a necessidade da implantação de um programa de avaliação freqüente desses equipamentos.

Os principais problemas encontrados foram: erro no volume de aplicação e na rotação de trabalho da tomada de força o trator para acionamento do pulverizador.

A manutenção e operação dos pulverizadores hidráulicos de barra esta sendo feita, de forma incorreta, tanto que $100 \%$ dos pulverizadores apresentaram ao menos um defeito.

A metodologia empregada foi adequada para a avaliação dos pulverizadores agrícolas, exceto para uniformidade de distribuição de calda na barra.

\section{Agradecimentos}

Ao CNPq, à FAPEMIG e à CAPES, pelo suporte financeiro que permitiu o desenvolvimento desta pesquisa.

\section{Referências Citadas}

Associação Brasileira de Normas Técnicas

1987a Norma NBR 9999: medição do nível do ruído, no posto de operação, de tratores e máquinas agrícolas. Rio de Janeiro.

Baldi, F.; Vieri, M.

1992 Controllo e certificazione delle macchine per la distribuzione dei Fitofarmaci. Macchine per la Distribuzione de Fitofarmaci, Bologna, v. 38, p. 17-32.

Balestrini, L.

2006 Mobile Inspection and Diagnosis Service of Sprayers in Resistance Prevention. Results Obtained Out of Inspections Performed by a Group of Producers During the 2004-2005 Season. Resistant Pest Management Newsletter, Michigan, v. 16, n. 1 , p. 5-7.

Bauer, F.C.; Raetano, C.G.; Pereira, F.A.R.

2006 Padrões de distribuição volumétrica de pontas de pulverização de jato plano 11002, com e sem indução de ar, sob diferentes espaçamentos e alturas. Engenharia Agrícola, Jaboticabal, v. 26, n. 2, p. 546-551.

Borghi, E.; Schreiner, R. C.; Ramos, H. H., Filho, A. P.; Yanai, K. 2003 Qualidade de pulverizadores utilizados em pequenas propriedades. Engenharia Agrícola, Jaboticabal, v. 23, n. 1, p. 113-121.

Freitas, F.C.L.; Teixeira, M.M.; Ferreira, L.R.; Ferreira, F.A.; Machado, A.F.L.; Viana, R.G.

2005 Distribuição volumétrica de pontas de pulverização turbo teejet 11002 em diferentes condições operacionais. Planta Daninha, Viçosa, v. 23, n. 1, p. 161-167.

Gandolfo, M. A.; Antuniassi, U.

2003 Inspeção periódica de pulverizadores agrícolas. Energia na Agricultura, Botucatu, v. 18, n. 2, p. 67-76.

$\mathrm{KOCH}, \mathrm{H}$.

1996 Periodic inspection of air-assisted sprayers. Workshop On Application Technology In Plant Protection, EPPO Bulletin, Braunschweig. v.26, p.79-86.
Magdalena, J.C., Di Prinzio, A.P.

1993 Servício de calibración de pulverizadoras frutícolas en Rio Negro y Neuquén. In: Congresso Argentino de Ingenieria Rural, 2, 1992, Córdoba. Anais... Córdoba: Maquinas y Tratores, p. 91-94.

Mialhe, L.G.

1996 Máquinas agrícolas: ensaios e certificação. Piracicaba: FEALQ.

Pergher, G.

2004 Field evaluation of a calibration method for air-assisted sprayers involving the use of a vertical patternator. Crop Protection, London, v. 23, p. 437-446.

Ramos, F.J.G.; Cortés, M.V.

2006 Inspección técnica de equipos para la aplicación de fitosanitarios. Vida Rural, Zaragoza, v. 227, p. 38-42.

Ramos, H.H.; Pio, L.C.

2003 Tecnologia de Aplicação de Produtos Fitossanitários. In: Zambolin, L.; Conceição, M. Z.; Santiago, T. O Que Os Engenheiros Agrônomos Devem Saber Para Orientar o Uso de Produtos Fitossanitários. 1. ed. Viçosa: Ed. UFV, cap. 5, p. 133-202.

Santos, S. R. dos; Maciel, A.J. da S.

2006 Proposta metodológica utilizando ferramentas de qualidade na avaliação do processo de pulverização. Engenharia Agrícola, Jaboticabal, v. 26, n. 2, p. 627-636, maio/ago.

Silveira, J.C.M.; Da, Filho, A.G.; Pereira, J.O.; Silva, S. De L.; Modolo, A.J.

2006 Avaliação qualitativa de pulverizadores da região de Cascavel, Estado do Paraná. Acta Scientiarum. Agronomy, Maringá, v. 28, n. 4, p. 569-573, Oct./Dec. 